\title{
A Printed Transition for Matching Improvement of SIW Horn Antennas
}

\author{
Marc Esquius-Morote, Benjamin Fuchs, Member, IEEE, Jean-François Zürcher, and Juan R. Mosig, Fellow, IEEE
}

\begin{abstract}
The substrate integrated waveguide (SIW) technology allows to construct several types of commonly used antennas in a planar way. However, frequency limitations associated to commercial substrates appear in the implementation of certain types of antennas, e.g., SIW horn antennas are not well matched when the substrate thickness is much smaller than the wavelength. A printed transition is proposed to overcome this problem. Differently from current solutions, no bulky elements are required allowing to maintain the most important features of this technology namely its compactness and ease of manufacturing. In order to quickly analyze and design the transition, both a coupled resonator and a transmission line models are developed, together with design guidelines. The proposed transition is designed to match a $\mathrm{H}$-plane SIW horn antenna built in a thin substrate (thickness $<\lambda_{0} / 10$ ) at different frequency bands at the Ku-band. Experimental results for 3 different transitions show that the matching characteristics are efficiently improved compared with the conventional SIW horn antenna and validates the proposed models.
\end{abstract}

Index Terms-Bandwidth, horn antenna, low profile, matching, printed transition, substrate integrated waveguide.

\section{INTRODUCTION}

$\mathbf{T}$ HE substrate integrated waveguide (SIW) technology is a very promising candidate for systems operating in the microwave and millimeter-wave region. The most significant advantage of SIW is the possibility to integrate all the components in the same substrate, including passive components (filters, couplers, etc.), active elements (oscillators, amplifiers, etc.) as well as antennas [1]-[8].

For applications requiring end-fire radiation, the most commonly used antenna is the SIW horn [9]-[11]. The design of an integrated H-plane sectoral horn antenna was proposed in [12]. However, its performances diminish when the substrate thickness is much smaller than the wavelength [13], [14]. This is also the case for other types of antennas such as the SIW slot antenna whose traditional formulae must be modified to fit the small thickness. At frequencies lower than $20 \mathrm{GHz}$, this is a frequently encountered situation when commercial substrates are used.

Manuscript received March 23, 2012; revised September 19, 2012; accepted November 15, 2012. Date of publication December 04, 2012; date of current version April 03, 2013. This work was supported in part by ArmaSuisse Science and Technology Federal Department of Defense, Civil Protection and Sport (DDPS) under the Contract Nr 041-18.

The authors are with the Laboratoire d'Electromagnétisme et d'Acoustique (LEMA), Ecole Polytechnique Fédérale de Lausanne (EPFL), CH-1015 Lausanne, Switzerland (e-mail: marc.esquiusmorote@epfl.ch).

Color versions of one or more of the figures in this paper are available online at http://ieeexplore.ieee.org.

Digital Object Identifier 10.1109/TAP.2012.2231923

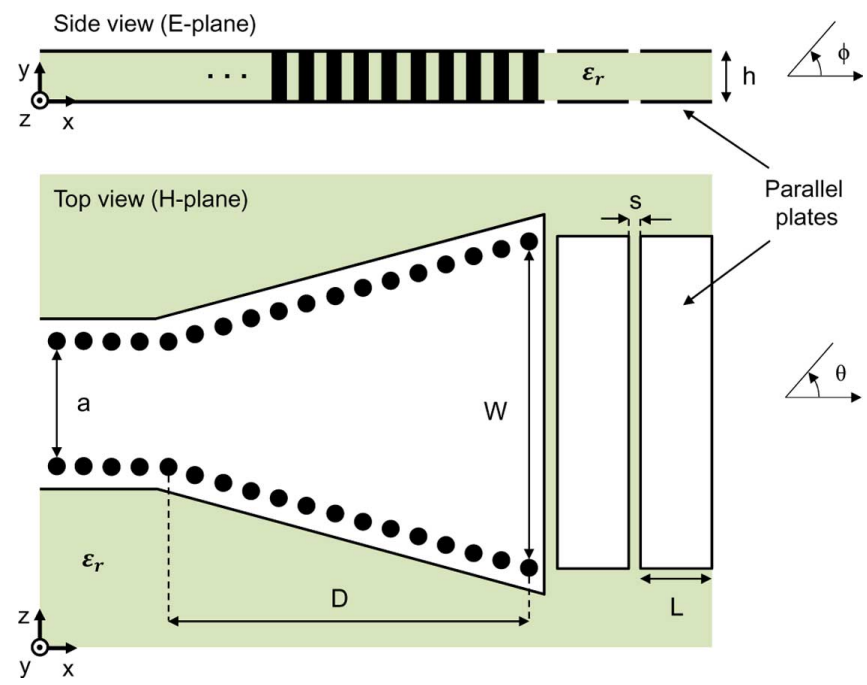

Fig. 1. Side and top view of a SIW horn with the proposed printed transition to the air.

Being able to overcome this thickness limitation would greatly extend the use of the SIW horn antennas. First, antennas working at lower frequencies could be built in commercial substrates. Second, other feeding techniques could substitute the typically used coaxial probe to completely integrate the antenna with other elements printed in the same substrate.

One of the main problems of SIW horns is the mismatch between the edge of the dielectric slab and the air which decreases the operational bandwidth. Several strategies have been developed to overcome this problem. Some of them require the use of non-printed elements [9], [15] making the building procedure more complicated. Solutions based on integrated dielectric lenses [10], [11] have also been proposed but their performances are limited by the thickness of the substrate. In the following, the terminology thin-substrate is used to describe a substrate with a thickness of less than $\lambda_{0} / 10$.

In this paper, a printed transition introduced in [16] for improving the matching between thin-substrate SIW horn antennas and the air is proposed. The transition is etched on the same dielectric slab as the antenna and does not require the use of nonprinted elements. This eases the manufacturing process while keeping the advantages of both compactness and integration of the SIW technology. A sectoral H-plane SIW horn with the proposed transition is represented in Fig. 1.

The paper is organized as follows. In Section II, the limitations of SIW horn antennas are explained and the proposed transition is described. Its working principle is analyzed in Section III where a coupled resonator model is derived to quickly predict its resonant frequencies. For more quantitative 


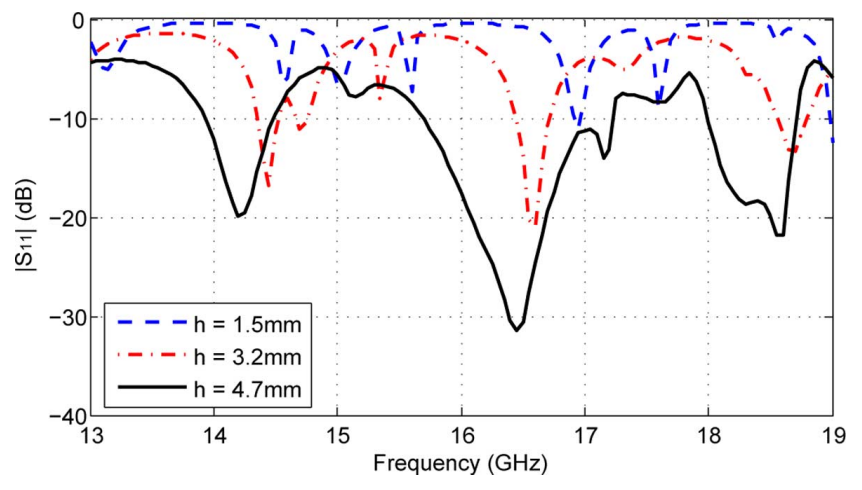

Fig. 2. $\left|S_{11}\right|$ comparison of a dielectric loaded SIW horn with a substrate of different thicknesses.

information about the reflection coefficient $\left|S_{11}\right|$, a transmission line (TL) model is developed in Section IV. Design guidelines are given in Section $\mathrm{V}$ and used to manufacture a prototype with different transitions. Finally, measurement results are provided in Section VI to prove both the improvements this printed transition brings in terms of matching and the validity of the proposed models.

\section{SIW Horn Limitations AND PROPOSED PRINTED TRANSITION}

The design rules for SIW horn antennas follow the same principles as normal horn antennas [17]. To ensure the single mode excitation of a H-plane SIW horn, the width of the feeding waveguide $a$ (see Fig. 1) should be such as $\lambda_{0} /\left(2 \sqrt{\varepsilon_{r}}\right)<a<$ $\lambda_{0} / \sqrt{\varepsilon_{r}}$ and the height $h$ (in this case the substrate thickness) smaller than $a$, where $\lambda_{0}$ is a wavelength in free space. Therefore, in order to only excite the fundamental mode $\mathrm{TE}_{10}, h$ can be as small as desired. However, when the dielectric slab becomes thinner, the mismatch between the horn aperture and the air increases which yields a poor matching and bandwidth.

To the author's best knowledge, the only currently available integrated solutions to improve the matching are based on dielectric lenses. This procedure provides good results in terms of matching and radiation as reported in [11]. Nevertheless, for substrates of small thicknesses (generally speaking $h<$ $\left.\lambda_{0} / 6\right)$, such solutions can not be applied because the effect of the lens is negligible.

To illustrate these limitations, the performances of a dielectric loaded SIW horn antenna with different thicknesses are compared. The horn is designed to work at $16.5 \mathrm{GHz}$ in a substrate of $\varepsilon_{r}=4.5$ that is extended $18 \mathrm{~mm}$ after the horn aperture to create the lens effect. According to the notations in Fig. 1, the dimensions of the horn are [mm]: $W=28, D=20$ and $a=9$. The $\left|S_{11}\right|$ of this horn with different substrate thicknesses $h$ was simulated using the 3-D EM simulator Ansys HFSS v13 (see Fig. 2). With a $h$ of $4.7 \mathrm{~mm}, \mathrm{a}-10 \mathrm{~dB}$ bandwidth of $10 \%$ is achieved around $16.5 \mathrm{GHz}$. However, as $h$ decreases, the bandwidth is greatly reduced until the point that, at $1.5 \mathrm{~mm}\left(h<\lambda_{0} / 10\right)$, the $\left|S_{11}\right|$ is practically flat presenting only poor narrow resonances.

The degradation of the reflection coefficient does not depend on the feeding system since the mismatch is located at the antenna-air interface. To smooth the impedance gap between the

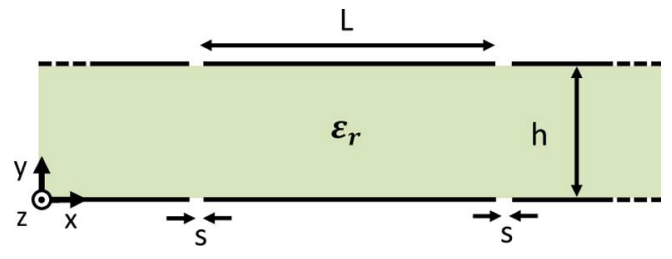

Fig. 3. Cutting view of the printed transition with the associated notations.

horn aperture and the air, a transition (represented in Fig. 1) printed on the same substrate as the horn is proposed. As detailed inSection III, a proper design of the printed transition (choice of the length $L$ and gap distance $s$ ) will match the horn at the desired frequency bands.

\section{COUPLED Resonator Model}

The proposed printed transition can be studied as a 2D structure where the width (z direction) is assumed to be infinite (see Fig. 3). To explain its working principle, the transition can be seen as a concatenation of blocks acting as resonators, each one being a parallel plate waveguide separated by gaps. The resonant frequency $f r_{1}$ of one block is

$$
f r_{1}=\frac{c}{2 L_{e q} \sqrt{\varepsilon_{r}}}
$$

where $L_{e q}$ is an equivalent length that is larger than $L$ to consider the effect of the fringing field. According to [18], $L_{e q}$ can be estimated by $L(1+0.7 h / L)$.

When two or more blocks are separated by a distance $s$, charges build up on the ends of the metallic plates, generating a capacitance which produces a shift in the resonant frequency $f r_{1}$. The physical explanation of this shift is that the coupling effect enhances and reduces the capability of a single block to store charge [19]. Thus, in the case of two blocks, a frequency higher $\left(f r_{2+}\right)$ and a frequency lower $\left(f r_{2-}\right)$ than $f r_{1}$ are generated.

In the theory of coupled resonators, a coupling factor $k_{2}$ is used to calculate $f r_{2 \pm}$. This factor is defined as the ratio between the capacitance generated by the coupling effect, $C_{C}$, and the capacitance of the original structure, $C_{0}$. Therefore, the new resonant frequencies can be calculated as follows:

$$
f r_{2 \pm}=\frac{f r_{1}}{\sqrt{1 \mp k_{2}}}
$$

Note that the presented structure differs from coupled microstrip resonators since there is no ground plane, i.e., there is a discontinuity (gap) at the ground plane. The presence of this gap slightly modifies the procedure used in the coupled resonators theory for calculating $f r_{i-}$ [19]. Actually, in this case, the gap enhances even more the capability to store charge of a single resonator. Therefore, the $f r_{i-}$ generated by the proposed structure are lower than the model predictions. However, in Section IV-C it is shown that the frequencies $f r_{i-}$ are of less interest than the $f r_{i+}$.

Let us now describe how to find the coupling factor $k_{i}$, i.e., $C_{C}$ and $C_{0}$, in the case of a structure of 2, 3 and $\mathrm{N}$ blocks. 


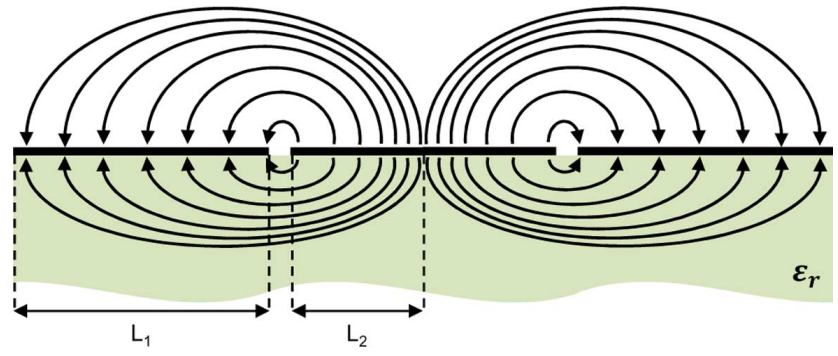

Fig. 4. Representation of the electric field distribution in the half upper part of a 3 block transition.

\section{A. Two Block Structure}

When two blocks are concatenated, the capacitances $C_{S}$ generated by the gaps at the top and bottom faces contribute to the coupling effect. Therefore, since both gaps are in series, $C_{C}$ equals $C_{S} / 2$.

The capacitance per unit of length $C_{S}$ can be obtained using the conformal mapping technique [20]. Thus, provided that $s / h \ll 1, C_{S}$ is given as follows:

$$
C_{S}\left(L_{1}, L_{2}\right)=\varepsilon_{0}\left(\varepsilon_{r}+1\right) \frac{K\left(\sqrt{1-p^{2}\left(L_{1}, L_{2}\right)}\right)}{K\left(p\left(L_{1}, L_{2}\right)\right)}
$$

with

$$
p\left(L_{1}, L_{2}\right)=\sqrt{\frac{1+\frac{L_{1}}{s}+\frac{L_{2}}{s}}{\left(1+\frac{L_{1}}{s}\right)\left(1+\frac{L_{2}}{s}\right)}}
$$

where $K()$ is the complete elliptic integral of the first kind. The lengths of the metallic plates at each side of the slot are noted as $L_{1}$ and $L_{2}$. In the current case, two equal blocks are concatenated meaning that $L_{1}=L_{2}=L$.

Concerning the capacitance of the original structure $C_{0}$, its value is twice the capacitance between the two metallic parallel plates of one block, $2 C_{P}$. The fringing fields effect must be considered to obtain an accurate result for $C_{P}$. This effect is only relevant at the exterior faces while it can be neglected at the junction between blocks. Following the approach presented in [21], the capacitance per unit of length $C_{P}$ can be estimated by

$$
C_{P} \simeq \frac{\varepsilon_{0} \varepsilon_{r} L}{h}\left(1+\frac{h}{\pi L} \ln \left(\frac{\pi L}{h}\right)\right) .
$$

Finally, the coupling factor for the case of 2 blocks is calculated as

$$
k_{2}=\frac{C_{C}}{C_{0}}=\frac{1}{2} \frac{C_{S}(L, L)}{2 C_{P}} .
$$

\section{B. $N$ Block Structure}

Several aspects should be taken into account when more than 2 blocks are concatenated. As an example, a structure of 3 blocks is now considered. A sketch of the electric field in the upper part of this structure is presented in Fig. 4, where it can be seen that now the central metallic plate contributes to generate two capacitances.
TABLE I

RESONANT FREQUENCIES FOR DIFFERENT TRANSITIONS ( $L=3.8 \mathrm{~mm}, h=1.5 \mathrm{~mm}, s=0.15 \mathrm{~mm}, \varepsilon_{r}=2.94$ )

\begin{tabular}{|c|c|c|}
\hline $\begin{array}{c}\text { Number } \\
\text { of blocks }\end{array}$ & $\begin{array}{c}\text { Full-wave } \\
\text { simulations }(\mathrm{GHz})\end{array}$ & $\begin{array}{c}\text { Coupled Resonator } \\
\text { Model }(\mathrm{GHz})\end{array}$ \\
\hline 1 & 17.95 & 18.03 \\
\hline 2 & 15.75 & 16.61 \\
\cline { 2 - 3 } & 19.50 & 19.89 \\
\hline \multirow{3}{*}{3} & 15.25 & 16.21 \\
\cline { 2 - 3 } & 17.20 & 18.03 \\
\cline { 2 - 3 } & 20.40 & 20.63 \\
\hline
\end{tabular}

In order to obtain the coupling capacitance $C_{C}$, the new values for the different $C_{S}$ can be calculated by applying again the conformal mapping technique. Nevertheless, for $s / L \ll 1$, a good approximation for the capacitance of each gap is to apply (3) with $L_{1}=L$ and $L_{2}=L / 2$ as illustrated in Fig. 4. This approach can be also followed in the case of more blocks. It provides a physical insight on the behavior of the structure without the need to use the conformal mapping technique in each scenario.

Regarding the calculation of $C_{0}$, the exterior blocks in a 3 block structure are under the same conditions as the ones of a 2 block structure. Thus, their capacitance value is also $C_{P}$. However, the middle block has no exterior faces meaning that its capacitance is calculated neglecting the fringing fields effect. Therefore, its per unit length value is $C_{P 0}=\varepsilon_{0} \varepsilon_{r} L / h$.

From the previous reasoning, the coupling factor $k_{3}$ for the case of 3 blocks is

$$
k_{3}=\frac{C_{C}}{C_{0}}=\frac{1}{2} \frac{2 C_{S}\left(L, \frac{L}{2}\right)}{2 C_{P}+C_{P 0}} .
$$

The same reasoning can be extended to $\mathrm{N}$ blocks by adapting the values of $C_{S}$ and $C_{P}$ for each gap or parallel plate structure, respectively. The general expression of the coupling factor $k_{N}$ where $N \geq 3$ is

$$
k_{N}=\frac{1}{2} \frac{2 C_{S}\left(L, \frac{L}{2}\right)+(N-3) C_{S}\left(\frac{L}{2}, \frac{L}{2}\right)}{2 C_{P}+(N-2) C_{P 0}} .
$$

The intermediate frequencies that appear between the lowest and highest frequency are also obtained following the theory of coupled resonators [19]. However, it will be explained in Section $\mathrm{V}$ that it is practically not convenient to design a more than 3 block transition.

\section{Coupled Resonator Model versus Full-Wave Simulations}

To validate the Coupled Resonator model, the resonant frequencies of a printed transition composed of 1, 2 and 3 block are also computed using HFSS. The resonances above $f r_{1}$ are predicted with a very good accuracy (relative error $<2 \%$ ) as shown in Table I. As expected, the resonances below $f r_{1}$ are less well estimated $(\sim 6 \%)$.

This Coupled Resonator model allows for a quick prediction of the resonant frequencies of the proposed transition. When quantitative information about the $\left|S_{11}\right|$ is required, more aspects need to be considered: source and load impedances, propagating modes, radiation losses, etc. Therefore, a Transmission Line model is proposed in Section IV. 


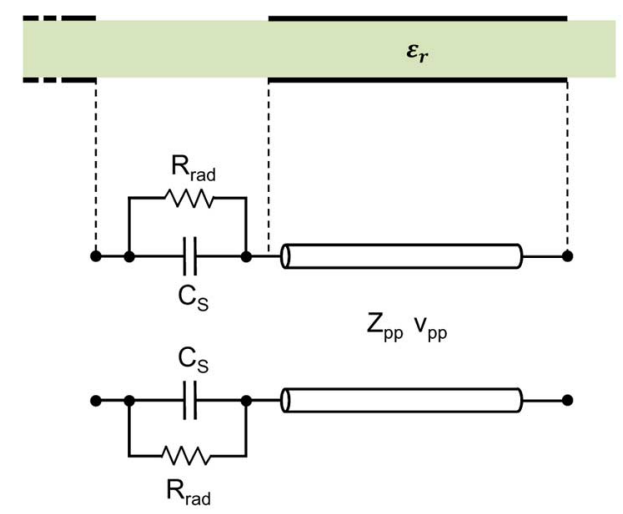

(a)

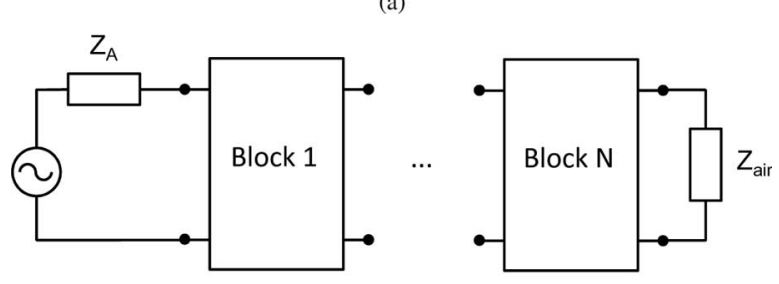

(b)

Fig. 5. Transmission Line model of the system: antenna, transition, air (a) TL model of one block (b) TL model of the whole system.

\section{TRANSMission Line Model}

A TL model is proposed to compute the $\left|S_{11}\right|$ of the printed transition. The transition is also decomposed into blocks, now taking into account the width $W$ in the $z$ direction defining a 3D problem. Each block is formed by a slot, modeled as a capacitance $C_{S}$ in parallel with a radiation resistance $R_{\text {rad }}$, and a parallel plate waveguide, defined by its characteristic impedance $Z_{p p}$ and propagation velocity $v_{p p}$. These blocks are then cascaded to model the full transition.

Note that the TL model of one block is asymmetric (see Fig. 5(a)). This has been chosen to accurately represent the transition structure which starts after the horn aperture with a gap and ends with a parallel plate open-ended termination.

The antenna (horn) is modeled as a generator with an equivalent impedance $Z_{A}$. Finally, a load $Z_{\text {air }}$ connected at the end of the TL model represents the outer boundary of the transition, i.e., the open-ended termination of the last parallel plate waveguide (see Fig. 5(b)).

The computation of the TL model parameters is now detailed.

\section{A. Block's Parameters}

One block is completely defined by four parameters: $C_{S}$, $R_{r a d}, Z_{p p}$ and $v_{p p}$.

The different values of $C_{S}$ are obtained using (3) with $W$ as length dimension. For more accurate results, the substrate thickness $h$ is also considered when using the conformal mapping technique [22].

The electric field distribution across the slot is nearly uniform. Therefore, the radiated power can be computed as the one of a uniform distribution aperture and, by assuming that the voltage between plates is $V=\left|E_{0}\right| s$, the radiation resistance $R_{\text {rad }}$ is given as follows:

$$
R_{r a d}=\frac{\left|E_{0}\right|^{2} s^{2}}{\oint_{S} W_{a v} d S}
$$

where $W_{a v}$ is the average power density of a uniform distribution aperture [17].

It is assumed that the main propagating mode in the parallel plates waveguide is the TEM mode. Therefore the following expressions are used:

$$
\begin{aligned}
& Z_{p p}=\sqrt{\frac{L_{p p}}{C_{p p}}} \\
& v_{p p}=\frac{1}{\sqrt{\varepsilon_{r p p} \varepsilon_{0} \mu_{0}}}
\end{aligned}
$$

where $L_{p p}$ and $C_{p p}$ are the inductance and capacitance in a TEM parallel plates waveguide and $\varepsilon_{r p p}$ is the effective relative permittivity between the rectangular parallel plates.

To obtain more accurate results, the values for $C_{p p}$ and $\varepsilon_{r p p}$ are computed taking into account the effect of the fringing fields. The expression of $C_{p p}$ depends on the position of the block following the same reasoning described for $C_{P}$ (4). Using the quasi-static approximation and figures presented in [23], $\varepsilon_{r \cdot p p}$ is computed as the quotient between the capacitance of the parallel plates filled with a dielectric substrate and filled with air.

\section{B. Antenna and Air Parameters}

A good approximation of the horn impedance where only the $\mathrm{TE}_{10}$ mode propagates is given by

$$
Z_{A}=\frac{k_{w g} \eta_{w g}}{\beta_{w g}} \frac{h}{W}
$$

where $k_{w g}$ is the wavenumber, $\eta_{w g}$ the wave impedance and $\beta_{w g}$ the propagation constant of the waveguide [24].

The open-ended termination of the last parallel plate waveguide is modeled with an air impedance $Z_{a i r}$ computed as

$$
Z_{\text {air }}=\frac{1}{W\left(g_{s}+j b_{s}\right)} .
$$

The real part of the air admittance $W g_{s}$ represents the radiation effect while the imaginary part $W b_{s}$ models the stored energy. Their expressions can be found in [25].

\section{TL Model versus Full-Wave Simulations}

A transition composed of several blocks is placed in front of a long horn in order to minimize the amplitude and phase error at the aperture. A commercial substrate Rogers RO 4350 $\left(\varepsilon_{r}=3.66, h=1.524 \mathrm{~mm}\right)$ is considered and the different dimensions [mm]: $L=3.6, s=0.2, W=20, D=50$. With these parameters, $f r_{1}$ is equal to $16.8 \mathrm{GHz}(1)$.

The $\left|S_{11}\right|$ comparison between HFSS simulations and the TL model for a transition of 2 and 3 blocks is presented in Fig. 6 . In both cases, a good agreement is achieved concerning the 


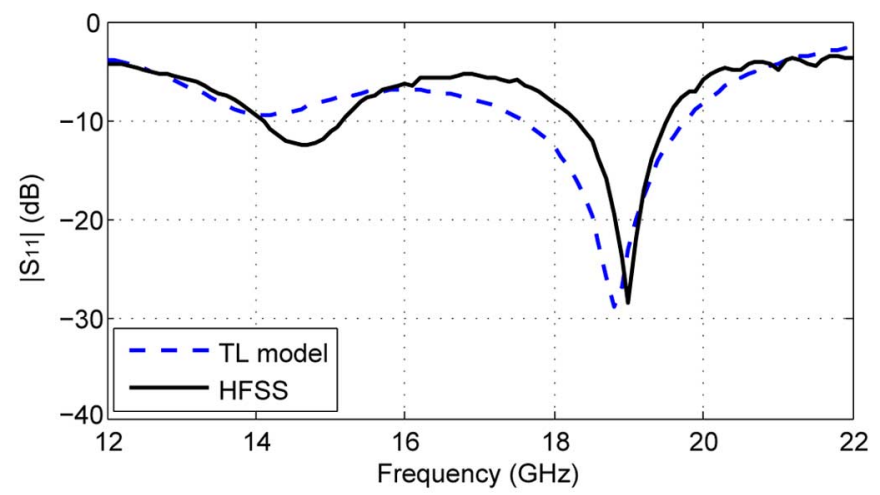

(a)

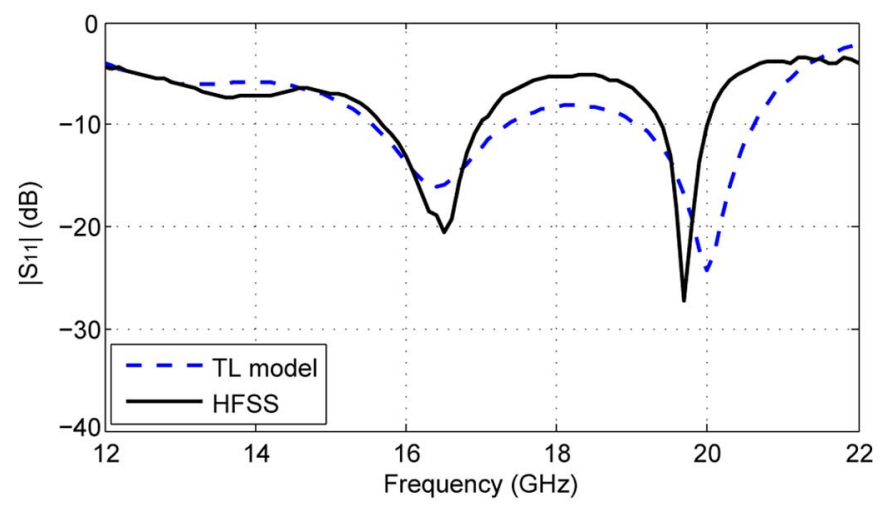

(b)

Fig. 6. $\left|S_{11}\right|$ comparison between HFSS and the TL model for a SIW horn with different transitions (a) Transition of 2 blocks (b) Transition of 3 blocks.

position and level of the resonances. Therefore, it is clearly demonstrated that fast and accurate preliminary results can be obtained using the TL model before performing final full-wave simulations.

As pointed out in Section III, the low resonances $f r_{i-}$ are due to the enhancement in the storage capability obtained with the coupling effect. It can be seen that, in general, these resonances present a poor matching $\left(\left|S_{11}\right|>-10 \mathrm{~dB}\right)$ being of less interest than the ones above $f r_{i+}$.

\section{Design Guidelines}

The steps to design a SIW horn with the proposed transition are the following:

1) With a given substrate ( $h$ and $\varepsilon_{r}$ ), the horn dimensions are chosen $(D, W$, and $a)$ to excite only the $\mathrm{TE}_{10}$ mode and to obtain an acceptable quadratic phase error at the horn aperture [17].

2) The number of blocks $(\mathrm{N})$ is chosen depending on the desired number of frequency bands. Then, (1)-(2) are used to compute the initial dimensions of the printed transition $(L$ and $s$ ). Afterwards, the TL model of Section IV is applied to further tune these dimensions.

According to the Coupled Resonator model described in Section III, it is in principle possible to design a transition working at any desired frequency. However, the following trends should be kept in mind when it comes to choose the values of $L, s, h$ and $\varepsilon_{r}$ :

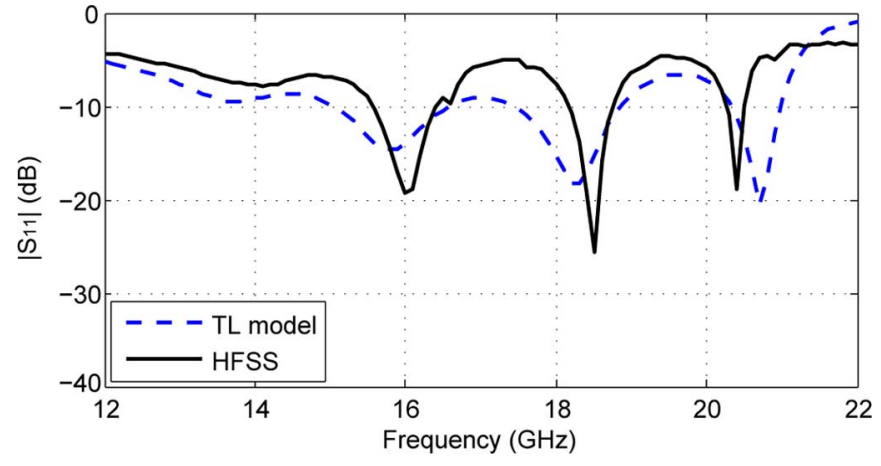

Fig. 7. $\left|S_{11}\right|$ comparison between HFSS and the TL model for a SIW horn with a 5 block transition.

- Increasing the ratio $h / L$ improves the matching and bandwidth but other modes might be excited.

- Reducing the ratio $s / L$ diminishes the radiation losses, but also the bandwidth is reduced.

- Increasing the value of $\varepsilon_{r}$ increases even more the antenna-air mismatch.

A reasonable trade-off is the following: $0.25<h / L<0.5$, $0.03<s / L<0.06, \varepsilon_{r}<5$. The accuracy of the two proposed models is good when the transition dimensions are within these boundaries. Considering the transition as a transformer between the antenna and the air impedances, a recommended starting point is to set to $L=0.4 \lambda_{0} / \sqrt{\varepsilon_{r}}$.

Regarding the choice of the number of blocks, it is recommended not to use more than 3. Adding more blocks increases indeed the radiation losses which leads to narrower and poorer resonances. To illustrate this effect, a transition of 5 blocks is placed in front of the SIW horn used for the comparison of Section IV-C. The simulated $\left|S_{11}\right|$ results with HFSS and the TL model are plotted in Fig. 7. As expected, even with the use of 5 blocks, only 3 narrow working frequency bands (relative bandwidth around $5 \%$ at $-10 \mathrm{~dB}$ ) are obtained.

\section{PRototype AND ExPERIMENTAL RESUlts}

A sectoral H-plane SIW horn antenna was built to show both the improvements this printed transition brings in terms of matching and the validity of the proposed models.

Using a commercial substrate Rogers TMM3 $\left(\varepsilon_{r}=\right.$ $3.27, h=1.91 \mathrm{~mm})$, this antenna alone presents a poor matching $\left(\left|S_{11}\right|>-10 \mathrm{~dB}\right)$ between 12 and $18 \mathrm{GHz}$ as shown in Fig. 9(a). Therefore, the proposed transition is implemented to match the SIW horn at different frequencies at this range.

Following the steps described in Section $\mathrm{V}$, the dimensions of the prototype are [mm]: $a=9, D=22, W=22, L=4.3$, $s=0.2$. In order to keep the radiation losses reasonably low, according to [2], the separation between vias and the via diameter were chosen to be $1.7 \mathrm{~mm}$ and $1 \mathrm{~mm}$ respectively. To feed the SIW horn with a microstrip line, a tapered microstrip-to-SIW transition was designed as described in [26].

The initial SIW horn prototype with a 3 block transition is shown in Fig. 8(a). As explained in Section III, the same horn can work at different frequency bands depending on the number of blocks at its aperture. In order to prove it, a diamond saw was used to cut one by one the exterior blocks of the transition. Thus, 


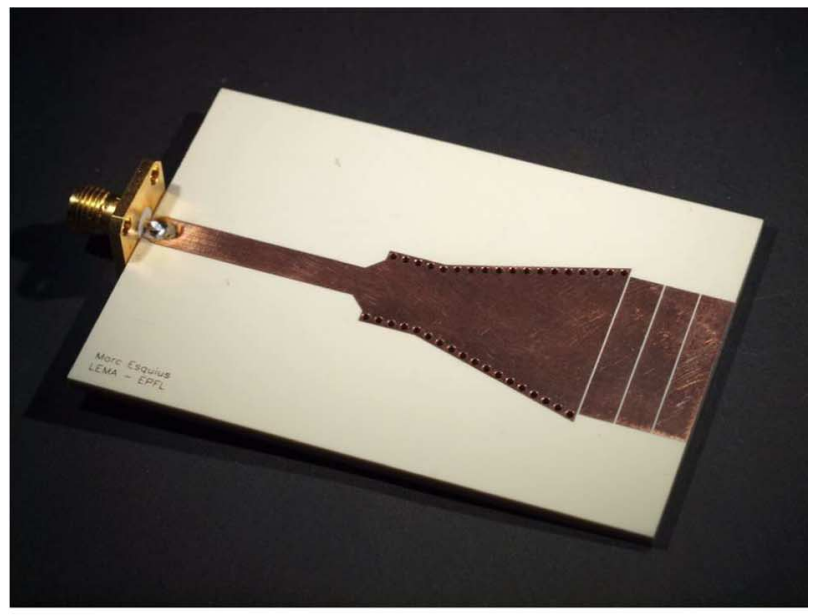

(a)

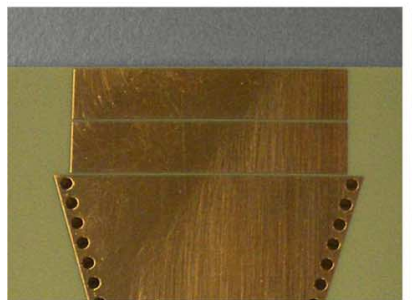

(b)

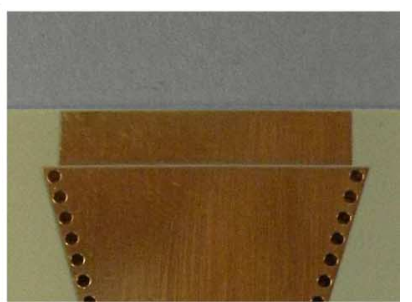

(c)

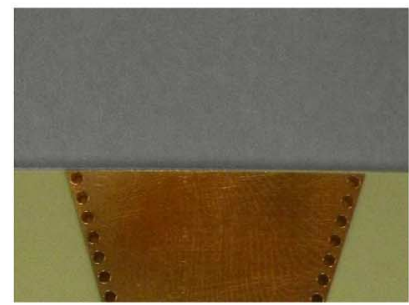

(d)

Fig. 8. Manufactured H-plane sectoral SIW horn prototype with a transition of different number of blocks (a) Original prototype with a 3 block transition (b) 2 block transition (c) 1 block transition (d) No transition.

as shown in Fig. 8, the same SIW horn with 3, 2, 1 and no block transition could be measured.

The $\left|S_{11}\right|$ comparison between the results of the coupled resonator model, the TL model, HFSS simulations and measurements are presented in Fig. 9(b)-(d). Clear working bands are obtained according to the printed transition number of blocks: $14.7 \mathrm{GHz}$ for 1 block, 12.2 and $16 \mathrm{GHz}$ for 2 blocks, 14.4 and 17.3 GHz for 3 blocks. As expected from Section IV-C, the lowest resonant frequency obtained with a 3 block transition has a poor matching being of less interest.

The measured and simulated positions of the resonant frequencies are in good agreement with the predictions of both proposed models. The measured bandwidths at $-10 \mathrm{~dB}$ are narrower than predicted by the TL model, but bands with a $10 \%$ relative bandwidth are obtained. Furthermore, a $4.5 \%-10 \mathrm{~dB}$ bandwidth is obtained with a two block transition at $12.2 \mathrm{GHz}$. At this frequency, the substrate thickness is thinner than $\lambda_{0} / 12$.

To obtain similar matching performances by the use dielectric lenses as proposed in [10], [11], a substrate thickness of around

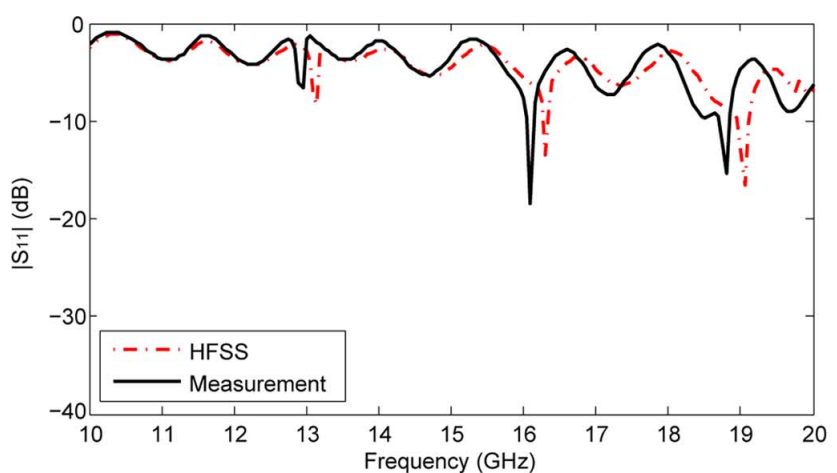

(a)

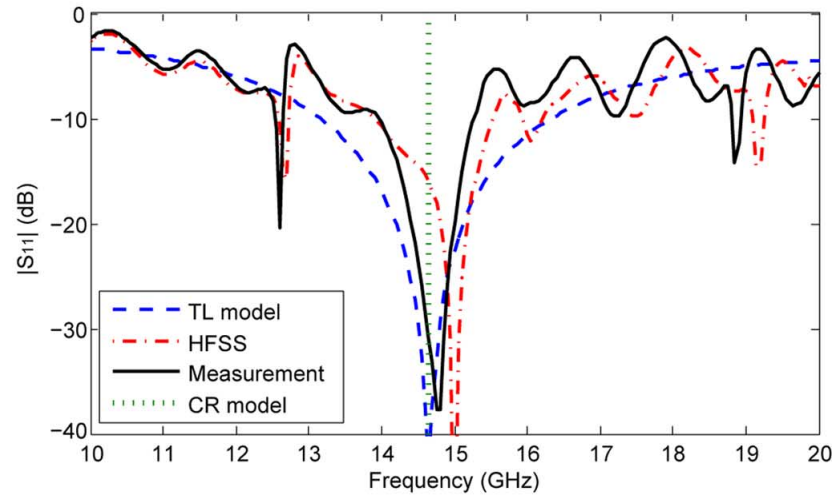

(b)

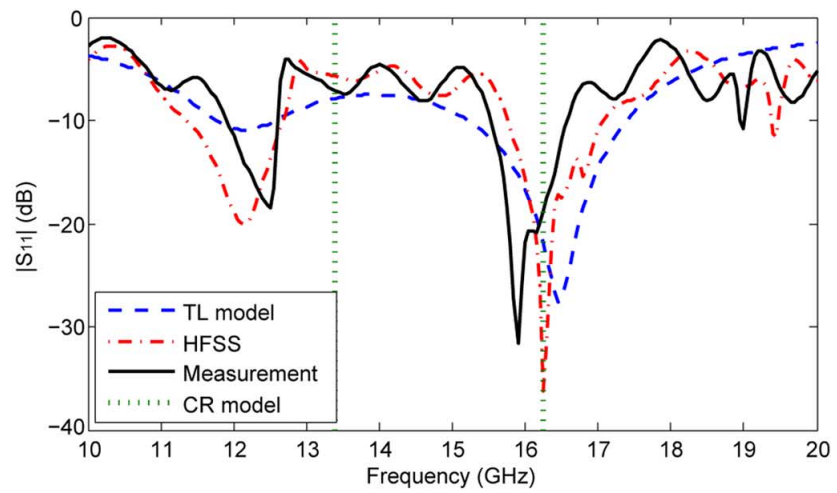

(c)

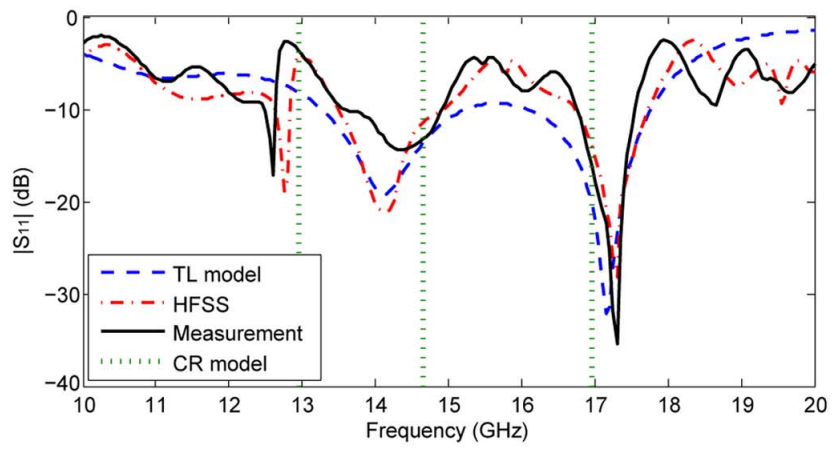

(d)

Fig. 9. $\left|S_{11}\right|$ of the SIW horn prototype with a transition of different number of blocks. Results for: Coupled Resonator (CR) model, TL model, HFSS and measurement (a) No transition (b) 1 block transition (c) 2 block transition (d) 3 block transition.

$\lambda_{0} / 5$ would be required. This means a $4 \mathrm{~mm}$ thick substrate instead of the $1.91 \mathrm{~mm}$ one used. 


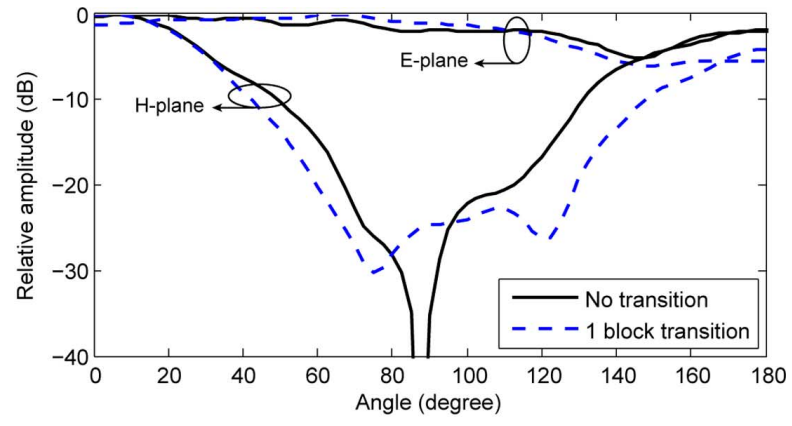

(a)

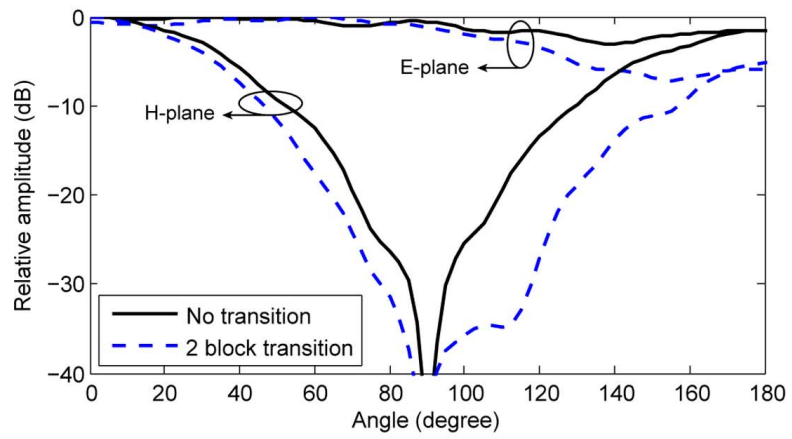

(b)

Fig. 10. Normalized radiation patterns of a SIW horn with different transitions. (a) 1 block transition at $14.7 \mathrm{GHz}$. (b) 2 block transition at $12.2 \mathrm{GHz}$.

\section{RADIATION CHARACTERISTICS}

The presented solution is focused on improving the reflection coefficient of H-plane SIW horn antennas. Depending on the application, a drawback of this type of antennas is the potentially high back radiation. The horn aperture practically behaves as a slot in an infinite ground plane with a front-to-back ratio (FTBR) close to unity.

The proposed printed transition does not significantly modify the radiation characteristics of a conventional SIW horn. The main radiating element of a SIW horn with the proposed transition is indeed the open-ended termination of the last parallel plates waveguide. Therefore, the differences in the radiation performances are mainly due to diffraction effects at the transition edges. In Fig. 10, the $\mathrm{E}$ and $\mathrm{H}$-plane radiation patterns of a SIW horn alone and with different transitions are compared. The presented patterns correspond to frequencies where $h<\lambda_{0} / 10$ and the horn is well matched by using a 1 and a 2 block transition. It is shown that the variation on the H-plane half power beamwidth is less than 5\% and the FTBR is improved by $3-4 \mathrm{~dB}$.

Modifications of the transition geometry are also under investigation to improve the radiation performances of SIW horn antennas.

\section{CONCLUSIONS}

A printed transition to improve the matching of H-plane SIW horn antennas has been proposed. Its aim is to reduce the mismatch between the horn aperture and the air at desired frequency bands. A coupled resonator and a transmission line models have been developed to analyze and design this transition. They allow for a quick prediction of its resonant frequencies as well as accurate preliminary results for the $\left|S_{11}\right|$. The validation of the models with full-wave simulations and measurements have been done in scenarios with different parameters to prove their wide validity range.

Design guidelines and recommendations to choose the transition dimensions are provided. They have been applied to manufacture a prototype of a thin-substrate H-plane SIW horn antenna which can work at different frequency bands at the $\mathrm{Ku}$-band. The overall antenna dimensions are very reasonable: horn length and width remain around $1.1 \lambda_{0}$ for a substrate thickness of $\lambda_{0} / 10$ and every transition strip only adds less than $0.25 \lambda_{0}$ to the total horn length. The experimental results have shown both the matching improvement brought by the transition and the validity of the proposed models.

It has been proved that the thickness of H-plane SIW horn antennas can be greatly reduced thanks to this transition. This allows the use of commercial substrates to built thin SIW horns working well below $20 \mathrm{GHz}$. Furthermore, a multi-band behavior can be achieved using a transition with different number of blocks. This extends the frequency range of horn antennas in SIW technology making them attractive for many other applications.

\section{REFERENCES}

[1] K. Wu, D. Deslandes, and Y. Cassivi, "The substrate integrated circuits-A new concept for high-frequency electronics and optoelectronics," in Proc. 6th Int. Conf. Telecommunications in Modern Satellite, Cable and Broadcasting Service, Oct. 2003, vol. 1.

[2] M. Bozzi, A. Georgiadis, and K. Wu, "Review of substrate-integrated waveguide circuits and antennas," Microw., Antennas Propag., IET, vol. 5, no. 8, pp. 909-920, Jun. 2011.

[3] Z.-C. Hao, W. Hong, J.-X. Chen, X.-P. Chen, and K. Wu, "Compact super-wide bandpass substrate integrated waveguide (SIW) filters," IEEE Trans. Microw. Theory Tech., vol. 53, no. 9, pp. 2968-2977, Sep. 2005.

[4] X. Chen, W. Hong, T. Cui, J. Chen, and K. Wu, "Substrate integrated waveguide (SIW) linear phase filter," IEEE Microw. Wireless. Compon. Lett., vol. 15, no. 11, pp. 787-789, Nov. 2005.

[5] Y. J. Cheng, W. Hong, and $\mathrm{K}$. Wu, "Millimeter-wave substrate integrated waveguide multibeam antenna based on the modified R-KR lens," in IEEE MTT-S Int. Microwave Symp. Dig., Jun. 2008, pp. 703-706.

[6] F. Giuppi, A. Georgiadis, A. Collado, M. Bozzi, and L. Perregrini, "Tunable SIW cavity backed active antenna oscillator," Electron. Lett., vol. 46, no. 15, pp. 1053-1055, 2010.

[7] J. Liu, D. Jackson, and Y. Long, "Substrate integrated waveguide (SIW) leaky-wave antenna with transverse slots," IEEE Trans. Antennas Propag., vol. 60, no. 1, pp. 20-29, Jan. 2012.

[8] R. Suga, H. Nakano, Y. Hirachi, J. Hirokawa, and M. Ando, "Costeffective $60-\mathrm{GHz}$ antenna package with end-fire radiation for wireless file-transfer system," IEEE Trans. Microw. Theory Tech., vol. 58, no. 12, pp. 3989-3995, Dec. 2010.

[9] Z. Li, X.-P. Chen, and K. Wu, "A surface mountable pyramidal horn antenna and transition to substrate integrated waveguide," in Proc. Int. Symp. Signals, Systems and Electronics, Feb. 2007, pp. 607-610.

[10] W. Che, B. Fu, P. Yao, Y. L. Chow, and E. K. N. Yung, "A compact substrate integrated waveguide $\mathrm{H}$-plane horn antenna with dielectric arc lens: Research articles," Int. J. RF Microw. Comput.-Aided Eng., vol. 17 , pp. 473-479, Sep. 2007.

[11] H. Wang, D.-G. Fang, B. Zhang, and W.-Q. Che, "Dielectric loaded substrate integrated waveguide (SIW) H-plane horn antennas," IEEE Trans. Antennas Propag., vol. 58, no. 3, pp. 640-647, Mar. 2010.

[12] Z. L. Li and K. Wu, "An new approach to integrated horn antenna," in Proc. Int. Symp. Antenna Technology and Applied Electromagnetics, Jun. 2004, pp. 535-538.

[13] C. Yeh, D. Yang, T. Liu, J. Fu, K. Chin, J. Cheng, H. Chiu, and C. Kao, "MMIC compatibility study of SIW H-plane horn antenna," in Proc. Int. Conf. Microwave and Millimeter Wave Technology (ICMMT), May 2010, pp. 933-936. 
[14] Y. J. Cheng and Y. Fan, "Millimeter-wave miniaturized substrate integrated multibeam antenna," IEEE Trans. Antennas Propag., vol. 59, no. 12 , pp. 4840-4844, Dec. 2011.

[15] M. Yousefbeiki, A. A. Domenech, J. R. Mosig, and C. A. Fernandes, "Ku-band dielectric-loaded SIW horn for vertically-polarized multisector antennas," in Proc. 6th Eur. Conf. Antennas and Propagation (EUCAP), Mar. 2012, pp. 2367-2371.

[16] M. E. Morote, B. Fuchs, and J. R. Mosig, "Analytical model of a printed transition for SIW antennas," in Proc. 6th Eur. Conf. Antennas and Propagation (EUCAP), Mar. 2012, pp. 414-417.

[17] C. A. Balanis, Antenna Theory-Analysis and Design, 3rd ed. New York, NY, USA: Wiley, 2005.

[18] K. Yazdandoost and D. Gharpure, "Simple formula for calculation of the resonant frequency of a rectangular microstrip antenna," in Proc. Sep. 1998, vol. 2, pp. 604-605.

[19] J.-S. Hong and M. Lancaster, Microstrip Filters for RF/Microwave Applications. New York, NY, USA: Wiley, 2001, ch. 8.2.

[20] S. Bedair, "Characteristics of some asymmetrical coupled transmission lines (short paper)," IEEE Trans. Microw. Theory Tech., vol. 32, no. 1, pp. 108-110, Jan. 1984.

[21] M. H. Bao, Handbook of Sensors and Actuators, 1st ed. New York, NY, USA: Elsevier, 2000, vol. 8.

[22] A. Abbosh and M. Bialkowski, "Deriving characteristics of the slotline using the conformal mapping technique," in Proc. 17th Int. Conf. Microwaves, Radar and Wireless Communications, May 2008, pp. 1-4.

[23] P. Benedek and P. Silvester, "Capacitance of parallel rectangular plates separated by a dielectric sheet," IEEE Trans. Microw. Theory Tech., vol. 20, no. 8, pp. 504-510, Aug. 1972.

[24] F. Ishihara and S. Iiguchi, "Equivalent characteristic impedance formula of waveguide and its applications," Electron. Commun. Japan - Part II: Electronics, vol. 75, pp. 54-66, 1992.

[25] J. James and P. Hall, Handbook of Microstrip Antenna 1. London, U.K.: IEE/Peregrinus, 1989, ch. 10.2 .

[26] D. Deslandes, "Design equations for tapered microstrip-to-substrate integrated waveguide transitions," in IEEE MTT-S Int. Microwave Symp. Dig., May 2010, pp. 704-707. IEEE 5th Int. Symp. Spread Spectrum Techniques and Applications,

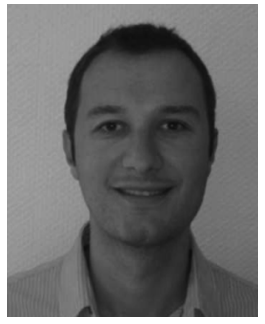

Benjamin Fuchs (S'06-M'08) received the Electronics Engineering degree and the M.S. degree in electronics from the National Institute of Applied Science (INSA) of Rennes, France, in 2004 and the $\mathrm{Ph} . \mathrm{D}$. degree from the University of Rennes 1 , in 2007.

In 2008, he was a postdoctoral research fellow at the Swiss Federal Institute of Technology (EPFL) Lausanne, Switzerland. In 2009, he joined the Institute of Electronics and Telecommunications of Rennes (IETR) as a Researcher at the Centre National de la Recherche Scientifique (CNRS). Since 2011, he is on leave at EPFL. His research interests include millimeter-wave antennas, focusing devices (lens antennas) and array synthesis methods.

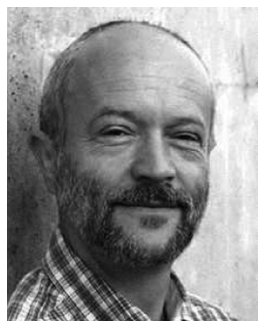

Jean-François Zürcher was born in Vevey, Switzerland, in 1951. He graduated with the degree of Electrical Engineer from Ecole Polytechnique Fédérale de Lausanne (Lausanne Institute of Technology), Lausanne, Switzerland, in 1974.

$\mathrm{He}$ is a permanent Scientific Associate with the Laboratoire d'Electromagnétisme et d'Acoustique EPFL, where he is the Manager of the Microwave Laboratory. His main interest lies in the domain of microstrip circuits and antennas. In 1988, he invented the SSFIP concept ("Strip Slot Foam Inverted Patch antenna"), which became a commercial product. He developed instrumentation and techniques for the measurement of near fields of planar structures and microwave materials measurement and imaging. He is currently working on millimeter-wave antennas design and measurement. $\mathrm{He}$ is the author or coauthor of more than 160 publications, chapters in books and papers presented at international conferences. He is one of the two authors of the book Broadband Patch Antennas (Norwood, MA, USA: Artech House, 1995). He holds nine patents.

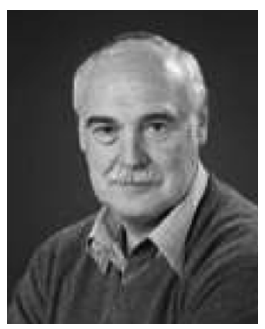

Juan R. Mosig (S'76-M'87-SM'94-F'99) was born in Cadiz, Spain. He received the Electrical Engineer degree in 1973 from Universidad Politécnica de Madrid, Madrid, Spain. In 1976, he joined the Laboratory of Electromagnetics and Acoustics (LEMA) at Ecole Polytechnique Fédérale de Lausanne (EPFL), Lausanne, Switzerland, from which he received the Ph.D. degree in 1983.

Since 1991, he has been a Professor at EPFL and since 2000 the Head of the EPFL Laboratory of Electromagnetics and Acoustics (LEMA). In 1984,

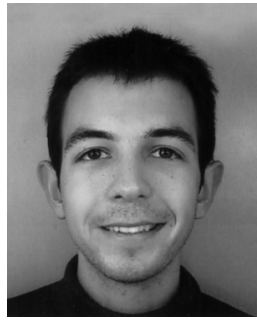

Marc Esquius-Morote was born in Barcelona, Spain, in 1986. He received the degrees in telecommunication and electronic engineering both from the Technical University of Catalonia (UPC), Barcelona, in 2010 and 2011, respectively. He is currently working towards the Ph.D. degree at the Laboratoire d'Electromagnétisme et d'Acoustique (LEMA), Ecole Polytechnique Fédérale de Lausanne (EPFL), Switzerland.

His research interests are focused in the design and realization of substrate integrated structures and conformal systems. During the last 2 years of his studies, he received a scholarship to work at the Antenna Lab group, UPC. he was a Visiting Research Associate at Rochester Institute of Technology, Rochester, NY, USA. He has also held scientific appointments at University of Rennes (France), University of Nice (France), Technical University of Denmark, Lyngby, and University of Colorado at Boulder, CO, USA since the 1980's and the Chair for the two last COST Antenna Actions 284 and IC0603 ASSIST (2003-2011). He is also a founding member \& General Chair of the European Association on Antennas and Propagation (EurAAP), owner of the EuCAP Conference series. He is the originator of a successful annual workshop, INTELECT, on Computational Electromagnetics. His research interests include electromagnetic theory, numerical methods and planar antennas. He has authored four chapters in books on microstrip antennas and circuits and over 120 reviewed papers.
Dr. Mosig has been the Swiss Delegate for European COST Antenna Actions 